Access
leading
journals in
your subject

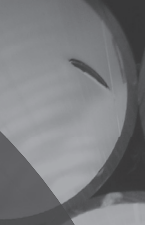


$S 680$ The structure of a turbulent line fountain G. R. Hunt, A. L. R. Debugne \& F. Ciriello

715 Enstrophy transport in swirl combustion
A. Kazbekov, K. Kumashiro \&

A. M. Steinberg

733 Statistics, plumes and azimuthally travelling waves in ultimate Taylor-Couette turbulent vortices

A. Froitzheim, R. Ezeta, S. G. Huisman, S. Merbold, C. Sun, D. Lohse \& C. Egbers

$S 766$ Spatial stability analysis of subsonic corrugated jets

F. C. Lajús, A. Sinha, A. V. G. Cavalieri, C. J. Deschamps \& T. Colonius

792 On kurtosis and extreme waves in crossing directional seas: a laboratory experiment

J. F. Luxmoore, S. Ilic \& N. Mori

818 Diffusiophoresis, Batchelor scale and effective Péclet numbers

F. Raynal \& R. Volk

830 High-speed shear-driven dynamos. Part 2. Numerical analysis

\section{K. Deguchi}

859 Self-similar turbulent vortex rings: interaction of propellant gases with blood backspatter and the transport of gunshot residue

P. M. Comiskey \& A. L. Yarin

881 Improved scaling laws for the shock-induced dispersal of a dense particle curtain

E. P. DeMauro, J. L. Wagner, L. J. DeChant, S. J. Beresh \& A. M. Turpin

896 Superharmonic instability of nonlinear travelling wave solutions in Hamiltonian systems

N. Sato \& M. Yamada

912 Interaction of viscous free-surface flows with topography

E. M. Hinton, A. J. Hogg \& H. E. Huppert

\section{JFM Rapids (online only)}

R1 Laboratory studies of Lagrangian transport by breaking surface waves

L. Lenain, N. Pizzo \& W. K. Melville

R2 Flight-crash events in superfluid turbulence P. Švančara \& M. La Mantia

R3 On oblique liquid curtains

S. J. Weinstein, D. S. Ross, K. J. Ruschak \& N. S. Barlow
939 Tilted drifting jets over a zonally sloped topography: effects of vanishing eddy viscosity H. Khatri \& P. Berloff

962 Droplets in homogeneous shear turbulence M. E. Rosti, Z. Ge, S. S. Jain, M. S. Dodd \& L. Brandt

$S 985$ Interaction between curvature-driven width oscillations and channel curvature in evolving meander bends

F. Monegaglia, M. Tubino \& G. Zolezzi

$S 1018$ On the scaling and topology of confined bluff-body flows

C. L. Ford \& P. M. Winroth

1041 Water drops bouncing off vertically vibrating textured surfaces

W. Wang, C. Ji, F. Lin, J. Zou \& S. Dorbolo

1052 Effective interfacial tension in flow-focusing of colloidal dispersions: 3-D numerical simulations and experiments

K. Gowda. V, C. Brouzet, T. Lefranc, L. D. Söderberg \& F. Lundell

1077 Einstein conjecture and resting-time statistics in the bed-load transport of monodispersed particles

L. Fraccarollo \& M. A. Hassan

1090 Thermal fluctuations in capillary thinning of thin liquid films

M. S. Shah, V. van Steijn, C. R. Kleijn \&

M. T. Kreutzer

$S 1108$ Combined measurement of velocity and temperature in liquid metal convection

T. Zürner, F. Schindler, T. Vogt, S. Eckert \& J. Schumacher

1129 Roughness effects in laminar channel flow Y. Liu, J. Li \& A. J. Smits

1146 Neutral stability curves of compressible Görtler flow generated by low-frequency free-stream vortical disturbances

S. Viaro \& P. Ricco

$S$ R4 Motion of Newtonian drops deposited on liquid-impregnated surfaces induced by vertical vibrations

P. Sartori, E. Guglielmin, D. Ferraro,

D. Filippi, A. Zaltron, M. Pierno \&

G. Mistura

$S$ indicates supplementary data or movies available online. 
1 Tuning heat transport via boundary layer topographies

K.-Q. Xia

5 Evolution of similarity lengths in anisotropic magnetohydrodynamic turbulence

R. Bandyopadhyay, W. H. Matthaeus, S. Oughton \& M. Wan

19 Mapping spheroid rotation modes in turbulent channel flow: effects of shear, turbulence and particle inertia

L. Zhao, N. R. Challabotla, H. I. Andersson \& E. A. Variano

55 Nonlinear hydroelastic waves on a linear shear current at finite depth

T. Gao, Z. Wang \& P. A. Milewski

$S 87$ Nonlinearly most dangerous disturbance for high-speed boundary-layer transition

R. Jahanbakhshi \& T. A. Zaki

122 Fully dispersive models for moving loads on ice sheets

\section{E. Dinvay, H. Kalisch \& E. I. Părău}

150 Optimal perturbations for controlling the growth of a Rayleigh-Taylor instability

\section{A. Kord \& J. Capecelatro}

186 A semi-Lagrangian direct-interaction closure of the spectra of isotropic variable-density turbulence D. J. Petty \& C. Pantano

237 Insights into the periodic gust response of airfoils

N. J. Wei, J. Kissing, T. T. B. Wester, S. Wegt, K. Schiffmann, S. Jakirlic, M. Hölling, J. Peinke \& C. Tropea

264 Numerical study of wedge-induced oblique detonations in unsteady flow

P. Yang, H. D. Ng \& H. Teng

288 Effects of nozzle-exit boundary-layer profile on the initial shear-layer instability, flow field and noise of subsonic jets

C. Bogey \& R. Sabatini

Contents continued on inside back cover.
326 Small-scale kinematics of two-phase flows: identifying relaxation processes in separatedand disperse-phase flow models

F. Drui, A. Larat, S. Kokh \& M. Massot

356 Evolution of thermally stratified turbulent open channel flow after removal of the heat source

M. P. Kirkpatrick, N. Williamson,

S. W. Armfield \& V. Zecevic

413 An extended linear shallow-water equation R. Porter

428 An improved model of near-inertial wave dynamics

O. Asselin \& W. R. Young

449 Inertial impedance of coalescence during collision of liquid drops

K. Sambath, V. Garg, S. S. Thete, H. J. Subramani \& O. A. Basaran

$S 481$ Formation and interaction of multiple secondary flow vortical structures in a curved pipe: transient and oscillatory flows

M. R. Najjari, C. Cox \& M. W. Plesniak

527 An improved Lagrangian model for the time evolution of nonlinear surface waves C.-A. Guérin, N. Desmars, S. T. Grilli, G. Ducrozet, Y. Perignon \& P. Ferrant

553 The minimum flow rate of electrosprays in the cone-jet mode

M. Gamero-Castaño \& M. Magnani

573 Feedback control of Marangoni convection in a thin film heated from below

A. E. Samoilova \& A. Nepomnyashchy

$S 591$ Self-channelisation and levee formation in monodisperse granular flows

F. M. Rocha, C. G. Johnson \&

J. M. N. T. Gray

$S 642$ Normal and oblique drop impact of yield stress fluids with thixotropic effects

C. M. Oishi, R. L. Thompson \& F. P. Martins 